\title{
Thrombin Generation in Vitreous and Subretinal Fluid of Patients with Retinal Detachment
}

\author{
Verena C. Mulder ${ }^{a}$ Jeroen Bastiaans ${ }^{b, c}$ Cornelis J.M. van Leuven ${ }^{d}$ \\ Jan C. van Meurs ${ }^{\mathrm{a}, \mathrm{e}}$ Cornelis Kluft $^{\mathrm{d}}$ \\ ${ }^{a}$ Rotterdam Ophthalmic Institute, Rotterdam Eye Hospital, Rotterdam, The Netherlands; \\ ${ }^{b}$ Department of Immunology, Erasmus Medical Centre, Rotterdam, The Netherlands; ' $D e p a r t m e n t ~ o f$ \\ Ophthalmology, Columbia University, New York, NY, USA; ${ }^{\mathrm{d}}$ Good Biomarker Sciences, Leiden, The Netherlands; \\ eDepartment of Ophthalmology, Erasmus Medical Centre, Rotterdam, The Netherlands
}

\section{Keywords}

Thrombin · Vitreous · Subretinal fluid · F1+2 - TAT .

a2-Macroglobulin · Hirudin · Proliferative vitreoretinopathy .

Retinal detachment

\begin{abstract}
Purpose: To measure prothrombin fragments $(\mathrm{F} 1+2)$ and thrombin-antithrombin complex (TAT) in vitreous and subretinal fluid (SRF) of rhegmatogenous retinal detachment (RRD) patients and to validate and further specify our earlier finding of increased thrombin activity in patients with proliferative vitreoretinopathy (PVR). Methods: F1+2 and TAT were measured in 31 vitreous and 16 SRF samples using the Enzygnost ${ }^{\circledR}$ immunoassays. Results: We found significant levels of $\mathrm{F} 1+2$ and TAT in the vitreous of all patients with RRD compared to patients with macular hole or macular pucker. However, there was no significant difference between patients who would develop PVR in the future, had established PVR, and patients with uncomplicated RRD both in vitreous concentrations of F1+2 (Kruskal-Wallis $p=0.963$ ) and TAT
\end{abstract}

$(p=0.516)$. Conclusion: The analysis of F1+2 and TAT confirmed significant thrombin generation in both vitreous and SRF of patients with RRD. An imbalance between the thrombin regulation mechanisms TAT and a2-macroglobulin possibly explains the difference from our previous findings.

(c) 2018 The Author(s)

Published by S. Karger AG, Basel

\section{Introduction}

Activation of the coagulation cascade has recently been identified as a potential factor in the development of proliferative vitreoretinopathy (PVR) [1]. We found significantly higher intravitreal thrombin concentrations in patients with established PVR and demonstrated that intravitreal thrombin stimulates retinal pigment epithelial cells to produce pro-inflammatory and pro-fibrotic mediators $[1,2]$.

To validate and further specify our earlier finding of increased thrombin activity in patients with PVR and to gain more insight into the different regulatory mecha-

\section{KARGER}

E-Mail karger@karger.com www.karger.com/oph
(C) 2018 The Author(s)

Published by S. Karger AG, Basel

Karger

Open access

This article is licensed under the Creative Commons AttributionNonCommercial-NoDerivatives 4.0 International License (CC BYNC-ND) (http://www.karger.com/Services/OpenAccessLicense). Usage and distribution for commercial purposes as well as any distribution of modified material requires written permission.
Verena C. Mulder, MSc, PharmD

Rotterdam Ophthalmic Institute

Schiedamse Vest 160D

NL-3011 BH Rotterdam (The Netherlands)

E-Mail v.mulder@ oogziekenhuis.nl 
nism of thrombin in ocular fluids, we explored two additional variables. The first is the concentration of the prothrombin activation fragment $(\mathrm{F} 1+2)$, which is a measure of the amount of thrombin generated from prothrombin. The second is the concentration of the thrombin-antithrombin complex (TAT) which is a measure of the amount of thrombin inhibited by antithrombin.

We further asked the question whether thrombin generation - marked by $\mathrm{F} 1+2$ and TAT - was different in patients with rhegmatogenous retinal detachment (RRD) who would develop PVR in the future or had established PVR, than in patients with uncomplicated RRD. Lastly, we discuss the implications for intervention with dabigatran, a reversible direct thrombin inhibitor.

\section{Methods}

\section{Sample Collection}

For the TAT and F1+2 analyses, we used vitreous fluid and subretinal fluid (SRF) samples from the Rotterdam Eye Hospital Biobank. Vitreous fluid or SRF are waste materials which are removed during a vitrectomy procedure or scleral buckling procedure, respectively. All patients gave their consent for the use of rest material for research.

Undiluted vitreous (1-1.5 mL) had been obtained at the start of vitrectomy before opening the infusion line. Undiluted SRF had been obtained by drainage through a 23-gauge needle mounted on a 2-mL syringe without a plunger [3]. Vitreous and SRF were immediately injected into Eppendorf tubes, provided with a unique number, and stored at $-80^{\circ} \mathrm{C}$. The location in the freezer and relevant information about the sample were noted on the registration form and later entered into an Access database (Microsoft Office ${ }^{\circledR}$ ).

We searched the biobank database for samples from patients who had undergone surgery for one of four conditions: RRD, PVR, a macular hole or a macular pucker. For patients with samples from RRD surgery, we checked their patient file to see whether they had undergone surgery for PVR later on. Samples from patients with a macular hole or macular pucker were included as controls. Samples from the biobank do not contain specific stabilisers. In vitro activation of coagulation seems negligible based on tests with vitreous and sodium citrate (unpublished data).

\section{F1+2 Analysis}

The quantification of the $\mathrm{F} 1+2$ prothrombin fragment was performed using the Enzygnost ${ }^{\circledR} \mathrm{F} 1+2$ monoclonal immunoassay (Siemens Healthcare Diagnostics Products, Marburg, Germany). According to the manufacturer's instructions, the samples were thawed using a water bath $\left(+37^{\circ} \mathrm{C}\right)$ for $10 \mathrm{~min}$. We diluted the sample 5 times - based on previous experience - using the sample buffer. Processing of samples and standard solution were performed according to the manufacturer's instructions [4].

Absorbance was measured at a wavelength of $450 \mathrm{~nm}$ using a spectrophotometer. The concentrations of $\mathrm{F} 1+2$ in the samples were derived from the constructed reference curve.

\section{TAT Analysis}

For the quantification of TAT, we used the Enzygnost ${ }^{\circledR}$ TAT micro immunoassay (Siemens Healthcare Diagnostics Products, Marburg, Germany). Based on previous experience the samples were diluted 10 times using the sample buffer. Further processing of samples and standard solution were performed according to the manufacturer's instructions [5].

Absorbance was measured at a wavelength of $492 \mathrm{~nm}$ using a spectrophotometer. The concentrations of TAT in the samples were derived from the constructed reference curve and were converted from $\mu \mathrm{g} / \mathrm{L}$ to $\mathrm{pmol} / \mathrm{L}$ using the molecular weight (MW) of the TAT complex of $96 \mathrm{kDa}$.

\section{Statistical Analysis}

Data were separately analysed for vitreous fluid and SRF. The results in vitreous fluid were analysed using the Kruskal-Wallis test. The results in SRF were analysed using the Mann-Whitney U test. A sub-analysis was performed for the three RRD groups. The relationship between F1+2 and TAT was tested using Spearman's correlation coefficient. A $p$ value $<0.05$ was considered significant. Analyses were performed with IBM SPSS statistics version 23 (IBM Corp., Armonk, NY, USA).

\section{Results}

A total of 47 samples were analysed. Distribution of samples across groups differed and was subjected to availability. We were able to obtain 31 vitreous samples and 16 SRF samples. SRF was only available for primary RRD surgeries and thus groups 3 and 4 .

\section{F1+2 Analysis}

Concentrations of $\mathrm{F} 1+2$ in vitreous differed significantly between the 5 groups $(p=0.008)$. The values in the two control groups were consistently low, while the concentration in the three RRD groups showed a large variation (Fig. 1). A Kruskal-Wallis sub-analysis in the three RRD groups could not detect a difference $(p=0.963)$. In addition, the $\mathrm{F} 1+2$ concentration in the SRF sample from the one patient who would, later on, develop PVR was not significantly different than the samples from the other RRD patients. The upper limit of quantification (ULQ) was $6,000 \mathrm{pmol} / \mathrm{L}$ and the lower limit of quantification (LLQ) was $35 \mathrm{pmol} / \mathrm{L}$ (both are shown as dotted lines). Values above the ULQ are shown as ULQ, values below the LLQ are shown as LLQ/2.

\section{TAT Analysis}

In Figure 2, the results are shown from the TAT measurements for both vitreous and SRF. Concentrations of TAT in vitreous differed significantly between the 5 groups $(p=0.002)$. The values in the two control groups 
Fig. 1. F1+2 measurements for both vitreous and subretinal fluid. The upper limit of quantification (ULQ) was $6,000 \mathrm{pmol} / \mathrm{L}$ and the lower limit of quantification (LLQ) was $35 \mathrm{pmol} / \mathrm{L}$ (both are shown as dotted lines). Values above the ULQ are shown as ULQ, values below the LLQ are shown as LLQ/2. MPCK, macular pucker; MHOLE, macular hole; RRD, rhegmatogenous retinal detachment; PVR, established proliferative vitreoretinopathy; RRD, later PVR, sample is from primary RRD surgery, patient developed PVR in later stage.
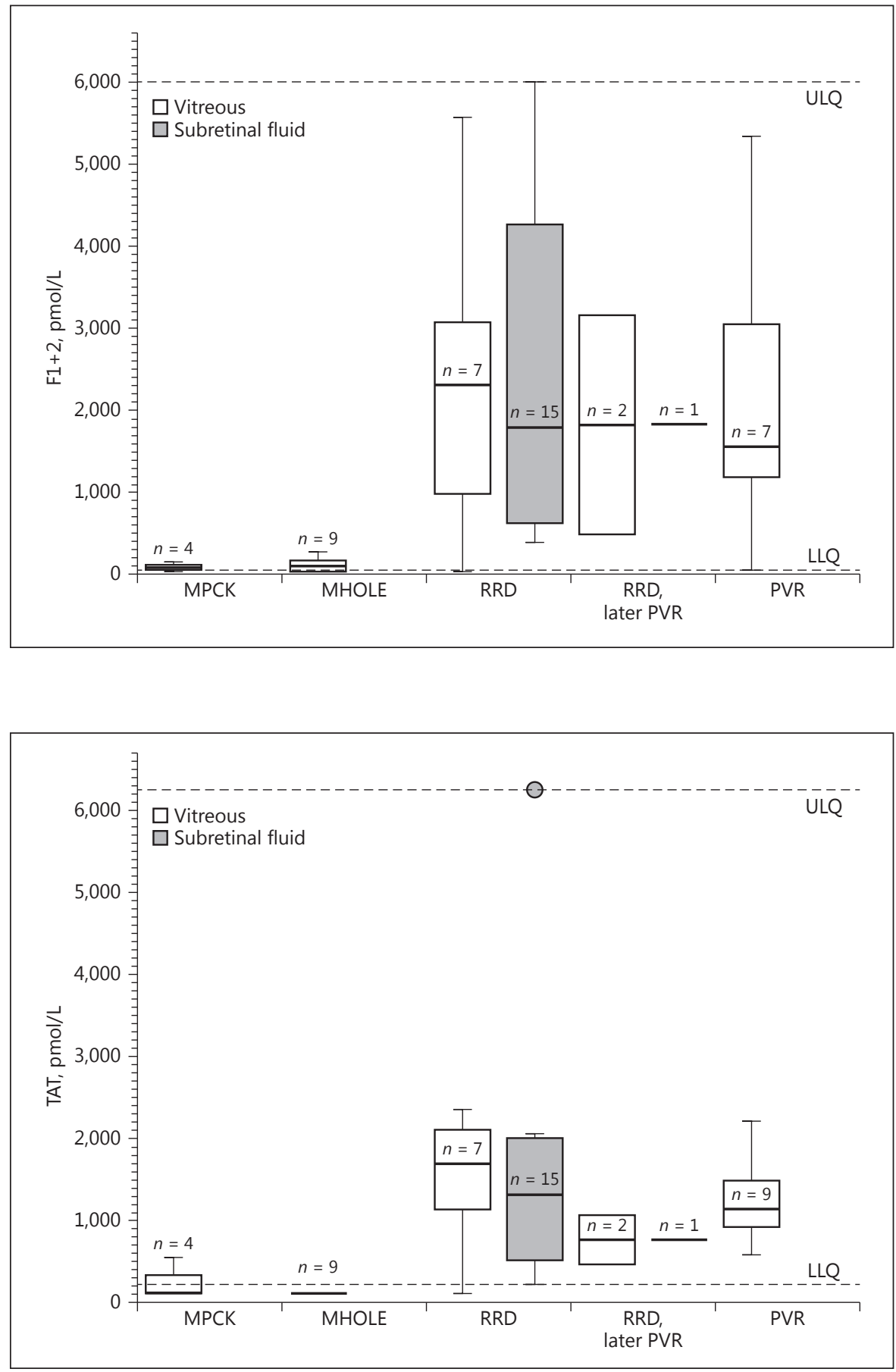

Fig. 2. TAT measurements for both vitreous and subretinal fluid. The upper limit of quantification (ULQ) was $6,250 \mathrm{pmol} / \mathrm{L}$ and the lower limit of quantification (LLQ) was $208 \mathrm{pmol} / \mathrm{L}$ (both are shown as dotted lines). Values above the ULQ are shown as ULQ, values below the LLQ are shown as LLQ/2. MPCK, macular pucker; MHOLE, macular hole; RRD, rhegmatogenous retinal detachment; PVR, established proliferative vitreoretinopathy; RRD, later PVR, sample is from primary RRD surgery, patient developed PVR in later stage.

were again consistently low. A Kruskal-Wallis sub-analysis in the three RRD groups could not detect a difference $(p=0.516)$. A difference in the TAT concentration in SRF between the two groups could not be detected. The ULQ was $6,250 \mathrm{pmol} / \mathrm{L}$ and the LLQ $208 \mathrm{pmol} / \mathrm{L}$ (both are shown as dotted lines). Values above the ULQ are shown as ULQ, values below the LLQ are shown as LLQ/2.

\section{Correlation between $\mathrm{F} 1+2$ and $T A T$}

The graph in Figure 3 shows the relationship between $\mathrm{F} 1+2$ and TAT. The production of thrombin marked as F1+2 was significantly related to the concentration of TAT both in vitreous $\left(r_{s}=0.84, p<0.001\right)$ and SRF $\left(r_{s}=\right.$ $0.93, p<0.001)$. The slope suggests that in vitreous $60 \%$ of thrombin was bound to antithrombin and in SRF 70\%. 


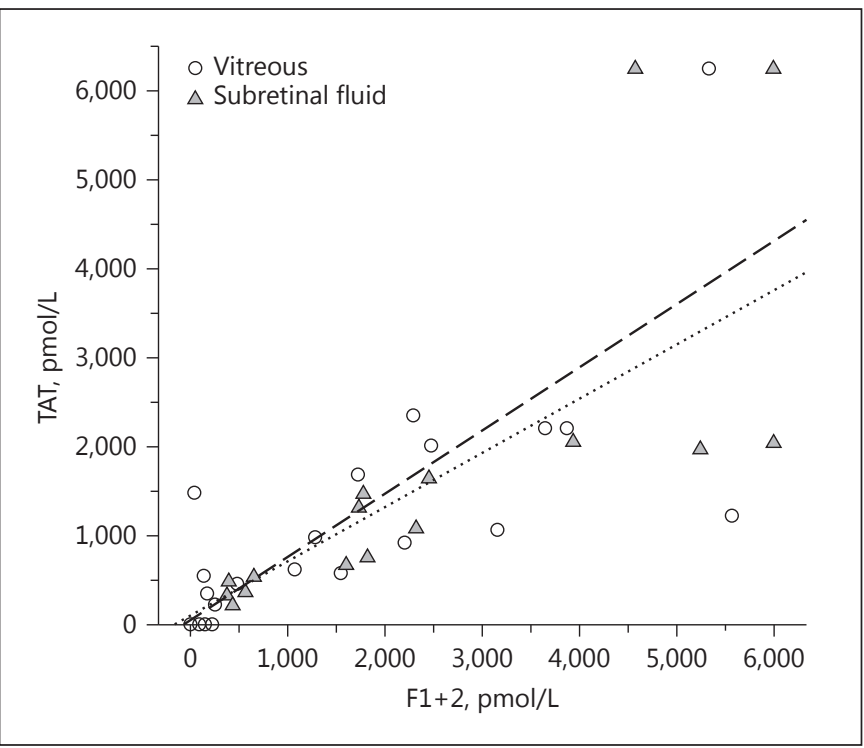

Fig. 3. Correlations of $\mathrm{F} 1+2$ and TAT values in subretinal fluid (triangles) and vitreous (circles). The dashed line represents the trend line of subretinal fluid and the dotted line the trend line of vitreous. Lower limit of quantification values are included as being 0 .

\section{Discussion}

These results of two indirect measures of thrombin formation confirm our previous results that significantly more thrombin was generated in vitreous fluids of patients with RRD in contrast to patients with a macular hole or macular pucker (Fig. 1,2) [1]. In contrast to our previous experiment, which showed significantly higher thrombin activity in established PVR, we could not detect a difference in F1+2 and TAT values between patients with uncomplicated RRD and patients with established PVR. These findings are important because our more direct measurements of thrombin activity were possibly not accurate and straightforward.

Interestingly, the median concentrations of $\mathrm{F} 1+2$ and TAT that we found in vitreous and SRF of RRD patients were much higher than in normal plasma. The median concentration of $\mathrm{F} 1+2$ was approximately 10 times higher than in plasma (normal range 69-229 $\mathrm{pmol} / \mathrm{L}$ ) and the median concentration of TAT was approximately 100 times higher than in plasma (normal range $20-40 \mathrm{pmol} / \mathrm{L}$ ) $[4,5]$. Our values of TAT were similar to those in vitreous recently reported by Ehrlich et al. [6]. These high values argue in favour of local thrombin activation.

Also the ratio of $\mathrm{F} 1+2$ versus TAT conforms with local thrombin activation. In plasma, TAT is cleared about 10 times faster by the liver from the circulation than $\mathrm{F} 1+2$ (8 min vs. $90 \mathrm{~min}$ ), which results in a difference in molar concentrations and a ratio of approximately 0.1 [7]. In the ocular fluids, the molar concentration ratio was closer to 1 (Fig. 3) indicating the formation of both $\mathrm{F} 1+2$ and TAT in the absence of differential clearance related to liver function in the circulation. The general higher levels in vitreous and SRF may be further explained by accumulation from slower clearance of the molecules from this compartment. When only the production determines the concentration, we expect a strong correlation between $\mathrm{F} 1+2$ and TAT. The Spearman correlation coefficient was indeed rather strong $\left(r_{s}=0.84\right.$ and $\left.r_{s}=0.93\right)$ and the slope suggests that thrombin is $60-70 \%$ bound to antithrombin (Fig. 3). The remaining $30-40 \%$ is possibly bound to other inhibitors such as $a 2$-macroglobulin ( $\alpha 2 \mathrm{M})$ or receptors.

What did we measure previously? In the previous experiments, we used the small thrombin-specific chromogenic substrate Tos-Gly-Pro-Arg-pNA (MW 662.7 Da) for the measurements of intravitreal thrombin activity [1]. Although the substrate is very well split by thrombin, other serine proteases such as plasma kallikrein and plasmin are also known to act on the substrate due to its small MW. Small synthetic substrates do not provide sufficient resemblance to the large natural substrates of thrombin, which was initially articulated by Gaffney et al. [8]. To distinguish between thrombin activity and activity of other enzymes, we duplicated the measurements with the addition of a very specific and irreversible inhibitor of thrombin: hirudin (MW $7 \mathrm{kDa}$ ) [1]. Specific thrombin activity in vitreous fluid was identified and quantified based on the difference in activity between vitreous fluid with and without hirudin. We assumed that what we recorded was only free thrombin activity.

At the time of those experiments, we were not aware of the possibility that thrombin bound to one of its inhibitors named a2M could still interact with small substrates and hirudin. In vivo, thrombin is mainly regulated by two inhibitors. The primary thrombin inhibitor is antithrombin, which forms the inactive TAT complex. The action of antithrombin can be increased significantly by the endogenous proteoglycan heparin [9]. The second inhibitor is a2M. a2M is a large plasma protein $(725 \mathrm{kDa})$ that upon binding undergoes a conformational change such that the $\alpha 2 \mathrm{M}$ folds around thrombin and partially shields its active site [10]. This prevents the cleavage of large substrates such as fibrinogen $(340 \mathrm{kDa})$ nearly completely but is not much effective for small substrates. The complex of $\alpha 2 \mathrm{M}$ with enzymes is rapidly cleared from the 
circulation; however, in a relatively enclosed compartment such as the eye or in vitro, a very slow remaining interaction of the complex with larger substrates (MW up to at least $20 \mathrm{kDa}$ ) remains possible and may continue to show thrombin activity $[10,11]$. Such slow interaction was shown for $\alpha 2 \mathrm{M}$-bound thrombin with hirudin on a time scale up to $4 \mathrm{~h}$ and for a2M-bound trypsin with soybean trypsin inhibitor (MW $20 \mathrm{kDa}$ ) on a time scale up to $40 \mathrm{~h}$ [11-13]. In the assay we used, we recorded the activity at different time points up to $10 \mathrm{~h}$ and based our analysis on the 8-hour values. Re-inspecting the time course of activity showed a progressive inhibition by hirudin similar to what was reported by Pochon and Steinbuch [11]. We now conclude that the previous report mainly concerned $\alpha 2 \mathrm{M}$-bound thrombin. The fact that thrombin is bound to $\alpha 2 \mathrm{M}$ does not change the hypothesis that thrombin plays a role in the development of PVR as the presence of the complex shows that thrombin had been formed. In addition, our previous experiment did show a significant increase in the production of CCL2, CXCL8, IL-6, and IL-12 in ARPE-19 cells after incubation with vitreous [1]. These effects were abolished by hirudin, which makes it unlikely that other enzymes than thrombin in the vitreous played a role.

The total amount of thrombin that has been locally active is probably better described by the concentration of $\mathrm{F} 1+2$ than the sum of TAT and our previous measurement of ( $\alpha 2 \mathrm{M}$-bound) thrombin. Although we now know that it is highly likely that all previously measured thrombin activity was bound to $\alpha 2 \mathrm{M}$, the exact quantification from that information is uncertain because of a question about the stoichiometry of the inhibition. We tested the hirudin sensitivity of the complex, but $\alpha 2 \mathrm{M}$ possesses two binding sites and binds one or two thrombin molecules. It is unknown whether hirudin is able to interact with both a2M-bound thrombin molecules. For trypsin, it was shown that only one of the two a2M-bound trypsin molecules interacted with soybean trypsin inhibitor [13]. On retrospect, we also observed residual activity seen after prolonged hirudin inhibition, which might not just be from other serine proteases but partly from a2M-bound thrombin not inhibited by hirudin. Using the hirudininhibited activity as a measure of thrombin activity - even after allowing enough incubation time - might therefore underestimate the thrombin activity.

We found significantly higher (what is now known to be) a2M-bound thrombin in patients with established PVR than in patients with uncomplicated RRD and patients who would later develop PVR. Surprisingly, we did not find this difference in $\mathrm{F} 1+2$ (formation marker) and

Thrombin Generation in Vitreous and SRF
TAT (thrombin portion bound by antithrombin) but found a remarkably broad range of values among the RRD patients. It will be of interest to know whether or not this range in degree of thrombin formation relates further to, for example, the size of the detachment or overall health of the patient. However, the limited sample size of this study rendered it not feasible to explore this.

Another possible explanation for this difference might be that not only the amount of thrombin generated causes PVR, but an imbalance in the different regulation mechanisms of thrombin determines whether or not thrombin causes the development of PVR. We can only speculate that the TAT portion is modulated by local heparins and that the $\alpha 2 \mathrm{M}$-bound thrombin portion is responsible for the cellular effects. After binding of $\alpha 2 \mathrm{M}$ to a proteinase, the resulting conformational change exposes a receptor recognition site on the a2M molecule. When this site is recognized by an $\alpha 2 \mathrm{M}$ receptor, mainly found on hepatocytes, macrophages, and fibroblasts, the complex is internalized and degraded $[10,14]$. Therefore, it is suggested that $22 \mathrm{M}$ may function as a scavenger for different peptide mediators in inflamed tissue and may constitute an important mechanism for the regulation and containment of inflammation [14]. In contrast, it was also demonstrated that $22 \mathrm{M}$ binds and modulates the biological activity of several cytokines [15]. Activated $\alpha 2 \mathrm{M}$ enhanced growth responses to TGF- $\beta 1$ in smooth muscle cells and also IL- 6 was shown to retain biological activity after binding to $22 \mathrm{M}[16,17]$. It may therefore also function as a carrier. An imbalance towards more $\alpha 2 \mathrm{M}$ would possibly show a less steep slope in the correlation graph of F1+2 and TAT; however, there were too few samples in the PVR groups to compare slopes.

In view of our hypothesis that the a2M-bound thrombin is the active compound in cellular reactions and that an imbalance between regulation mechanism plays a role, the question arises whether or not an artificial inhibitor of thrombin such as dabigatran changes the distribution of thrombin between antithrombin and $\alpha 2 \mathrm{M}$. In an in vitro experiment in plasma, we observed that the presence of dabigatran actively changed the distribution among inhibitors. With the same concentration of thrombin, we found an increasing concentration of TAT with increasing concentrations of dabigatran (unpublished data). This is considered to be a consequence of the difference in affinity of antithrombin and $\alpha 2 \mathrm{M}$ for thrombin. The addition of dabigatran, a reversible inhibitor that competes with antithrombin and $\alpha 2 \mathrm{M}$ for thrombin, results in favouring the formation of complexes with the inhibitor with the highest affinity (antithrombin), which would 
be advantageous if the goal is to have less $\alpha 2 \mathrm{M}$-bound thrombin.

What are the implications of these results for our proposed therapy with the small direct thrombin inhibitor dabigatran? The median F1+2 concentration of 2,000 $\mathrm{pmol} / \mathrm{L}$ among the RRD groups suggests a four times larger thrombin activity than we previously reported [1]. A recalculation, however, of the dabigatran concentration needed to inhibit this amount of thrombin revealed no large increase. For an $80 \%$ inhibition, a concentration of $9.4 \mathrm{ng} / \mathrm{mL}$ would be needed [18].

At what point in time and how long thrombin has been active remains unclear. However, even when this amount of thrombin would be active all at once, the above-calculated concentration of dabigatran would be able to inhibit both free and a2M-bound thrombin [19]. An additional aim of dabigatran treatment in the prevention of PVR could be reducing a $2 \mathrm{M}$-thrombin formation by shifting the balance towards inactive TAT.

In conclusion, the analysis of F1+2 and TAT confirmed significant thrombin generation in both vitreous and SRF of patients with RRD. Vitreous dabigatran concentrations after oral intake would suffice to inhibit this amount of thrombin activity. Although we found a significant difference between uncomplicated RRD and PVR in thrombin activity in our previous study, we could not detect a difference in the formation of thrombin and inactivation via TAT. Possibly, additional factors such as overall health and imbalance between the regulatory mechanisms of thrombin play a role in steering an RRD towards PVR. A larger study including ASA scores, medication use, peroperative information, and analysis of F1+2, TAT, (a2M-bound) thrombin activity, and their ratios might give more insight into these factors.

\section{Acknowledgements}

This research was funded by Stichting Wetenschappelijk Onderzoek het Oogziekenhuis - Prof. Dr. H.J. Flieringa, and the research at GBS was supported by the company.

\section{Disclosure Statement}

C.J.M.v.L. and C.K. are employees of GBS.

\section{References}

1 Bastiaans J, van Meurs JC, Mulder VC, Nagtzaam NM, Smits-te Nijenhuis M, Dufour-van den Goorbergh DC, van Hagen PM, Hooijkaas H, Dik WA: The role of thrombin in proliferative vitreoretinopathy. Invest Ophthalmol Vis Sci 2014;55:4659-4666.

-2 Bastiaans J, van Meurs JC, van Holten-Neelen C, Nagtzaam NM, van Hagen PM, Chambers RC, Hooijkaas H, Dik WA: Thrombin induces epithelial-mesenchymal transition and collagen production by retinal pigment epithelial cells via autocrine PDGF-receptor signaling. Invest Ophthalmol Vis Sci 2013;54: 8306-8314.

3 Weijtens O, Schoemaker RC, Lentjes EG, Romijn FP, Cohen AF, van Meurs JC: Dexamethasone concentration in the subretinal fluid after a subconjunctival injection, a peribulbar injection, or an oral dose. Ophthalmology 2000;107:1932-1938.

4 Anonymous: Enzygnost ${ }^{\circledR}$ F1+2 Monoclonal; instruction manual. Marburg, Germany, Siemens Healthcare Diagnostics Products, 2010.

5 Anonymous: Enzygnost ${ }^{\circledR}$ TAT micro; instruction manual. Marburg, Germany, Siemens Healthcare Diagnostics Products, 2008.
6 Ehrlich R, Zahavi A, Axer-Siegel R, Budnik I, Dreznik A, Dahbash M, Nisgav Y, Megiddo E, Kenet G, Weinberger D, Livnat T: Correlation between interleukin-6 and thrombin-antithrombin III complex levels in retinal diseases. Curr Eye Res 2017;42:1269-1272.

7 Chandler WL, Velan T: Estimating the rate of thrombin and fibrin generation in vivo during cardiopulmonary bypass. Blood 2003;101: 4355-4362.

8 Gaffney PJ, Miller-Andersson M, Kirkwood TB: Unreliability of chromogenic substrates for assay of the clotting activity of thrombin. Haemostasis 1978;7:109-112.

-9 Bjork I, Lindahl U: Mechanism of the anticoagulant action of heparin. Mol Cell Biochem 1982;48:161-182.

10 Travis J, Salvesen GS: Human plasma proteinase inhibitors. Annu Rev Biochem 1983;52: 655-709.

11 Pochon F, Steinbuch M: Interaction of alpha 2-macroglobulin-bound thrombin with hirudin. FEBS Lett 1984;177:109-111.

12 Wang D, Wu K, Feinman RD: The reaction of alpha 2-macroglobulin-bound trypsin with soybean trypsin inhibitor. J Biol Chem 1981; 256:10934-10940.

13 Bieth JG, Tourbez-Perrin M, Pochon F: Inhibition of alpha 2-macroglobulin-bound trypsin by soybean trypsin inhibitor. J Biol Chem 1981;256:7954-7957.
14 Rehman AA, Ahsan H, Khan FH: Alpha2-macroglobulin: a physiological guardian. J Cell Physiol 2013;228:1665-1675.

15 Bonner JC, Goodell AL, Lasky JA, Hoffman MR: Reversible binding of platelet-derived growth factor- $\mathrm{AA},-\mathrm{AB}$, and $-\mathrm{BB}$ isoforms to a similar site on the "slow" and "fast" conformations of alpha 2-macroglobulin. J Biol Chem 1992;267:12837-12844.

16 Stouffer GA, LaMarre J, Gonias SL, Owens GK: Activated alpha 2-macroglobulin and transforming growth factor-beta 1 induce a synergistic smooth muscle cell proliferative response. J Biol Chem 1993;268:1834018344.

17 Matsuda T, Hirano T, Nagasawa S, Kishimoto T: Identification of alpha 2-macroglobulin as a carrier protein for IL-6. J Immunol 1989; 142:148-152.

18 Mulder VC, Kluft C, van Meurs JC: Vitreous and subretinal fluid concentrations of orally administered dabigatran in patients with rhegmatogenous retinal detachment. Acta Ophthalmol 2016;94:663-667.

19 Wagenvoord RJ, Deinum J, Elg M, Hemker HC: The paradoxical stimulation by a reversible thrombin inhibitor of thrombin generation in plasma measured with thrombinography is caused by alpha-macroglobulinthrombin. J Thromb Haemost 2010;8: 1281-1289. 